\title{
Low angle tracking with symmetric beam pattern monopulse based on uniform circular array
}

\author{
Min Guo ${ }^{1, a, *}$, Wenzheng Wu ${ }^{1}$, Shilin Yang ${ }^{1}$, Zhanshan Sun ${ }^{1}$ and Yunqi Fu ${ }^{1}$ \\ ${ }^{1}$ School of Electronic Science and Engineering, National University of Defense Technology, \\ Changsha, China \\ a guomin100@126.com
}

Keywords: Monopulse radar, low angle tracking, uniform circular array, symmetrical monopulse ratio.

\begin{abstract}
In this paper, the problem of low angle tracking is studied on the viewpoint of improving monopulse. We applied the traditional symmetrical monopulse ratio method to the uniform circular array. By dividing the circular antenna array into two equivalent sub arrays, a symmetrical monopulse ratio is formed, which could overcome the difficulty of angle measurement caused by multipath and meet the requirements of low angle tracking. The monopulse response curve and the precision of angle measurement are analysed by simulative experiments. Negative factors including thermal noise, asymmetrical multipath, signal-to-noise ratio (SNR) and reflection coefficient are discussed intensively with respect to the accuracy of our method.
\end{abstract}

\section{Introduction}

Symmetrical monopulse ratio method is an improved monopulse technique widely used in low angle tracking [1-3]. By redesigning the sum channel and difference channel, the monopulse response curve can be symmetrical with respect to the bore sight of array, and then the influence of multipath reflection signals can be eliminated. Similarly, the uniform circular array is common in the missile, aircraft and other equipments for its structural characteristics. In addition, it can be designed to be conformal, which makes it more advantageous over other geometries.

In recent years, there are numberous researches on symmetric monopulse elevation measurement algorithm. [4] proposed a method of symmetric error pattern. By designing two asymmetric sum channel and difference channel, the symmetrical angle curve against the target and the mirror target is formed, so that the influence of the specular reflection signal on the monopulse ratio is eliminated. The key of this method is to determine the beam directly. If the beam direction is not coincident with the angle bisector of the target and the mirror image [5], new error will be introduced. In [6], the monopulse response curve is obtained by the design of two asymmetric sum channel and difference channel. In [3], the symmetrical monopulse response curve is obtained by solving the optimal symmetric beam of the linear array antenna, and the minimum angle error is obtained. 
Some other elevation estimation methods based on array antenna are proposed in [7], but the engineering implementation is complex.

In this paper, the symmetrical difference pattern monopulse technique is applied to the uniform circular array. Firstly, we divide the antenna array into two subarrays, sum pattern and difference pattern are formed based on two subarrays individually. Then, the symmetric beam can be obtained by multiplying the monopulse response curve of the two subarrays. In this way, the influence of multipath on the angle measurement is overcomed.

\section{Basic principle}

\subsection{The basic principle of symmetric beam method}

For a monopulse system, the sum pattern is an even function, the difference pattern is an odd function, so the monopulse response carve is an odd function. Under the condition of multipath reflection, the ratio of difference pattern to sum pattern is a complex value. This is mainly affected by the reflection coefficient, which varies with the reflection surface and is always a complex value.

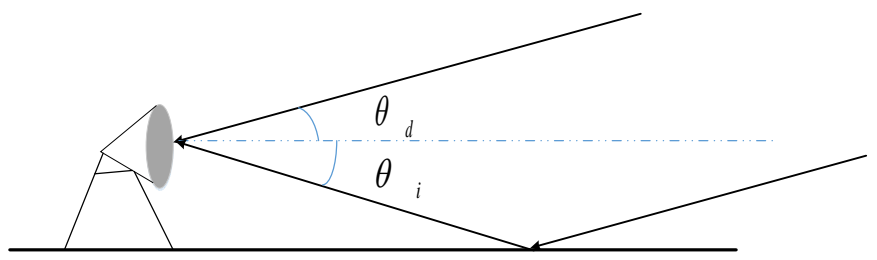

Figure.1 Schematic diagram of multipath

Supposing that the diffuse emission energy is ignorable, the specular reflection is mainly considered. In general the targets are far enough from the radar, thus the direct ray and the specular ray (before reflection) are parallel. As shown in Figure.1, the incident angles of the direct signal and specular signal are equal $\theta_{d}=-\theta_{i}$. The ratio of the difference pattern to the sum pattern is:

$$
\frac{\Delta}{\Sigma}=\frac{V_{\Delta}\left(\theta_{d}\right) A_{d}(t)+V_{\Delta}\left(\theta_{i}\right) A_{i}(t)}{V_{\Sigma}\left(\theta_{d}\right) A_{d}(t)+V_{\Sigma}\left(\theta_{i}\right) A_{i}(t)}=\frac{V_{\Delta}\left(\theta_{d}\right)+\rho V_{\Delta}\left(\theta_{i}\right)}{V_{\Sigma}\left(\theta_{d}\right)+\rho V_{\Sigma}\left(\theta_{i}\right)}=\frac{V_{\Delta}\left(\theta_{d}\right)+\rho V_{\Delta}\left(-\theta_{d}\right)}{V_{\Sigma}\left(\theta_{d}\right)+\rho V_{\Sigma}\left(-\theta_{d}\right)}=\frac{V_{\Delta}\left(\theta_{d}\right)-\rho V_{\Delta}\left(\theta_{d}\right)}{V_{\Sigma}\left(\theta_{d}\right)+\rho V_{\Sigma}\left(\theta_{d}\right)}=\frac{V_{\Delta}\left(\theta_{d}\right)(1-\rho)}{V_{\Sigma}\left(\theta_{d}\right)(1+\rho)}
$$

Where $V_{\Sigma}(\theta)$ and $V_{\Delta}(\theta)$ are the voltage of difference beam and sum beam, respectively. $A_{d}(t)$ and $A_{i}(t)$ are the time domain complex envelope of the direct signal and specular signal, respectively. $\rho=A_{i}(t) / A_{d}(t)$ is the complex reflection coefficient. Equation (1) shows that the ratio of the monopulse system is not only related to the elevation angle, but also the function of the complex reflection coefficient. If the complex reflection coefficient is unknown, the target elevation angle cannot be obtained.

If the beam is designed to make the difference pattern, namely $V_{\Delta}\left(\theta_{d}\right)=V_{\Delta}\left(-\theta_{d}\right)$, to be even, the monopulse ratio can be written as:

$$
\frac{\Delta}{\Sigma}=\frac{V_{\Delta}\left(\theta_{d}\right) A_{d}(t)+V_{\Delta}\left(\theta_{i}\right) A_{i}(t)}{V_{\Sigma}\left(\theta_{d}\right) A_{d}(t)+V_{\Sigma}\left(\theta_{i}\right) A_{i}(t)}=\frac{V_{\Delta}\left(\theta_{d}\right)+\rho V_{\Delta}\left(\theta_{i}\right)}{V_{\Sigma}\left(\theta_{d}\right)+\rho V_{\Sigma}\left(\theta_{i}\right)}=\frac{V_{\Delta}\left(\theta_{d}\right)+\rho V_{\Delta}\left(-\theta_{d}\right)}{V_{\Sigma}\left(\theta_{d}\right)+\rho V_{\Sigma}\left(-\theta_{d}\right)}=\frac{V_{\Delta}\left(\theta_{d}\right)+\rho V_{\Delta}\left(\theta_{d}\right)}{V_{\Sigma}\left(\theta_{d}\right)+\rho V_{\Sigma}\left(\theta_{d}\right)}=\frac{V_{\Delta}\left(\theta_{d}\right)}{V_{\Sigma}\left(\theta_{d}\right)}
$$

Equation (2) shows that the ratio has no relation with the complex reflection coefficient, and the influence of the specular multipath is eliminated. Remarkably, if $\rho=-1$, that is, the sum channel is 0 , then the elevation angle of target cannot be measured. 


\subsection{The principle of dividing eight circular- antenna arrays into subarrays to form symmetric beam.}

To make the ratio of the difference beam and the sum beam an even function, we divide the uniform circular array into two subarrays, each of which could be seen as a conventional monopulse system. As shown in Fig.2, where the antenna 1, 3, 5 and 7 belong to the monopulse system 1, the antenna 2, 4, 6 and 8 belong to the monopulse system 2. Two monopulse response curves could be got based on two sub arrays. Then, we multiply these two response curve and obtain a new angle curve, which is symmetric. In this way, negative influence caused by specular multipath is eliminated.

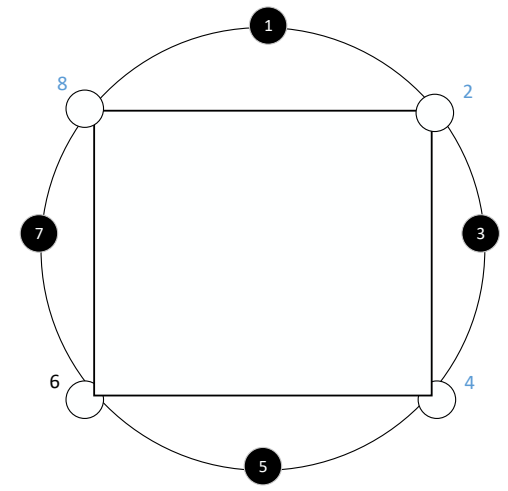

Figure.2 Antenna array partition structure

For monopulse system 1, the response curve is:

$$
\frac{\Delta_{1}}{\Sigma_{1}}=\frac{V_{\Delta 1}\left(\theta_{d 1}\right) A_{d 1}(t)+V_{\Delta 1}\left(\theta_{i 1}\right) A_{i 1}(t)}{V_{\Sigma 1}\left(\theta_{d 1}\right) A_{d 1}(t)+V_{\Sigma 1}\left(\theta_{i}\right) A_{i 1}(t)}=\frac{V_{\Delta 1}\left(\theta_{d 1}\right)(1-\rho)}{V_{\Sigma 1}\left(\theta_{d 1}\right)(1+\rho)}
$$

Similarly, for monopulse system 2 :

$$
\frac{\Delta_{2}}{\Sigma_{2}}=\frac{V_{\Delta 2}\left(\theta_{d 2}\right) A_{d 2}(t)+V_{\Delta 2}\left(\theta_{i 2}\right) A_{i 2}(t)}{V_{\Sigma 2}\left(\theta_{d 2}\right) A_{d 2}(t)+V_{\Sigma 2}\left(\theta_{i 2}\right) A_{i 2}(t)}=\frac{V_{\Delta 2}\left(\theta_{d 2}\right)(1-\rho)}{V_{\Sigma 2}\left(\theta_{d 2}\right)(1+\rho)}
$$

By multiplying response curves of monopulse system 1 and 2, we obtain the response curve of entire system:

$$
\frac{\Delta}{\Sigma}=\frac{\Delta_{1}}{\Sigma_{1}} \cdot \frac{\Delta_{2}}{\Sigma_{2}}=\frac{V_{\Delta 1}\left(\theta_{d 1}\right) A_{d 1}(t)}{V_{\Sigma 1}\left(\theta_{d 1}\right) A_{d 1}(t)} \cdot \frac{V_{\Delta 2}\left(\theta_{d 2}\right) A_{d 2}(t)}{V_{\Sigma 2}\left(\theta_{d 2}\right) A_{d 2}(t)}=\frac{V_{\Delta 1}\left(\theta_{d 1}\right) V_{\Delta 2}\left(\theta_{d 2}\right)}{V_{\Sigma 1}\left(\theta_{d 1}\right) V_{\Sigma 2}\left(\theta_{d 2}\right)}
$$

Both $\Delta_{1} / \Sigma_{1}$ and $\Delta_{2} / \Sigma_{2}$ are odd functions, so $\Delta / \Sigma$ is an even function. Considering the influence of specular reflection, $\Delta / \Sigma$ can be written as:

$$
\begin{aligned}
\frac{\Delta}{\Sigma} & =\frac{\Delta_{1}}{\Sigma_{1}} \cdot \frac{\Delta_{2}}{\Sigma_{2}}=\frac{V_{\Delta 1}\left(\theta_{d 1}\right) V_{\Delta 2}\left(\theta_{d 2}\right)+V_{\Delta 1}\left(\theta_{i 1}\right) V_{\Delta 2}\left(\theta_{i 2}\right)}{V_{\Sigma 1}\left(\theta_{d 1}\right) V_{\Sigma 2}\left(\theta_{d 2}\right)+V_{\Sigma 1}\left(\theta_{i}\right) V_{\Sigma 2}\left(\theta_{i 2}\right)}=\frac{V_{\Delta 1}\left(\theta_{d 1}\right) V_{\Delta 2}\left(\theta_{d 2}\right)+V_{\Delta 1}\left(-\theta_{i 1}\right) V_{\Delta 2}\left(-\theta_{i 2}\right)}{V_{\Sigma 1}\left(\theta_{d 1}\right) V_{\Sigma 2}\left(\theta_{d 2}\right)+V_{\Sigma 1}\left(-\theta_{i}\right) V_{\Sigma 2}\left(-\theta_{i 2}\right)} \\
& =\frac{V_{\Delta 1}\left(\theta_{d 1}\right) V_{\Delta 2}\left(\theta_{d 2}\right)+\rho V_{\Delta 1}\left(\theta_{d 1}\right) \rho V_{\Delta 2}\left(\theta_{d 2}\right)}{V_{\Sigma 1}\left(\theta_{d 1}\right) V_{\Sigma 2}\left(\theta_{d 2}\right)+\rho V_{\Delta 1}\left(\theta_{d 1}\right) \rho V_{\Delta 2}\left(\theta_{d 2}\right)}=\frac{V_{\Delta 1}\left(\theta_{d 1}\right) V_{\Delta 2}\left(\theta_{d 2}\right)\left(1+\rho^{2}\right)}{V_{\Sigma 1}\left(\theta_{d 1}\right) V_{\Sigma 2}\left(\theta_{d 2}\right)\left(1+\rho^{2}\right)}=\frac{V_{\Delta 1}\left(\theta_{d 1}\right) V_{\Delta 2}\left(\theta_{d 2}\right)}{V_{\Sigma 1}\left(\theta_{d 1}\right) V_{\Sigma 2}\left(\theta_{d 2}\right)}
\end{aligned}
$$

Obviously, the response curve of our method is independent of the complex reflection coefficient.

\section{Simulation Results and analysis}

In order to demonstrate how the sum pattern and difference pattern is formed, we assume that the single element antenna pattern is $\sin \varphi / \varphi$, the incident angle of direct echo is denoted as $\varphi_{0}$. The sum pattern can be expressed as: 


$$
\Sigma=2\left[\frac{\sin \left(\varphi-\varphi_{0}\right)}{\left(\varphi-\varphi_{0}\right)}+\frac{\sin \left(\varphi+\varphi_{0}\right)}{\left(\varphi+\varphi_{0}\right)}\right]
$$

The difference pattern can be expressed as:

$$
\Delta=2\left[\frac{\sin \left(\varphi-\varphi_{0}\right)}{\left(\varphi-\varphi_{0}\right)}-\frac{\sin \left(\varphi+\varphi_{0}\right)}{\left(\varphi+\varphi_{0}\right)}\right]
$$

In this simulation, the incidence of monopulse system 1 is set to 15 degrees, and the incidence of monopulse system 2 is set to 30 degrees, and the response carve of the monopulse system 1 and 2 are shown in Fig.3. For the monopulse system 1 and 2, the angle carve are odd function. In the -30 to 30 degrees, they can be approximated by a linear function, the result of fitting is shown in Fig. 4 .
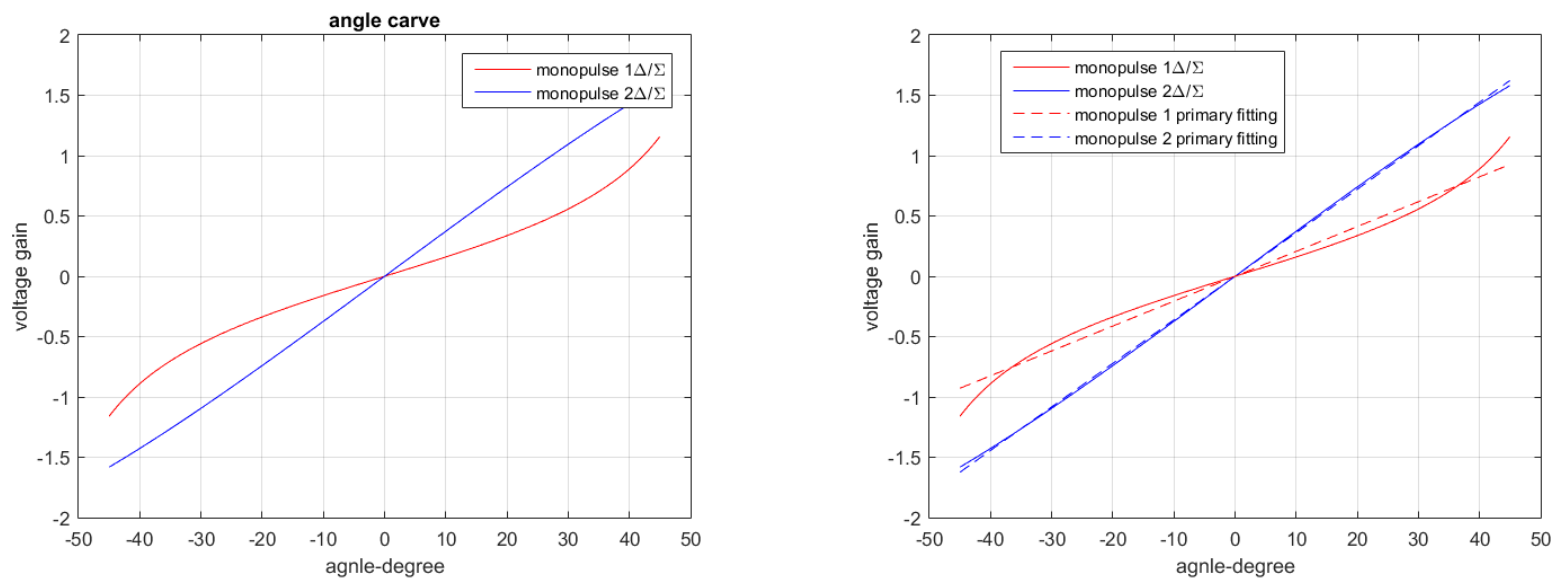

Figure. 3 The angle carve of the monopulse system 1 and 2 Figure. 4 The result of fitting by a quadratic function

The results of fitting can be approximately expressed as:

$$
\frac{\Delta_{1}}{\Sigma_{1}}=k_{1} \theta \quad \frac{\Delta_{2}}{\Sigma_{2}}=k_{2} \theta
$$

For the symmetrical beam pattern monopulse based on eight- antenna circular array, the angle carve is shown in Figure. 5 and can be expressed as:

$$
\frac{\Delta}{\Sigma}=\frac{\Delta_{1}}{\Sigma_{1}} \cdot \frac{\Delta_{2}}{\Sigma_{2}}=k_{1} k_{2} \theta^{2}
$$

The derivation of symmetric beam angle curve is as:

$$
d \theta=\frac{1}{2 k_{1} k_{2} \theta} d \frac{\Delta}{\Sigma}
$$

Equation (11) indicate that the angle accuracy is related to the elevation angle of the target. At the beam direction, the monopulse is not sensitive to the elevation angle of the target, and the angle measurement accuracy is the worst. With the increase of the elevation angle of the target, the measurement accuracy is improved. This is the characteristic of symmetrical beam pattern monopulse. In addition, $k_{1}$ and $k_{2}$ also have an influence on the accuracy of angle measurement. The bigger the value, the higher the accuracy of angle measurement. 


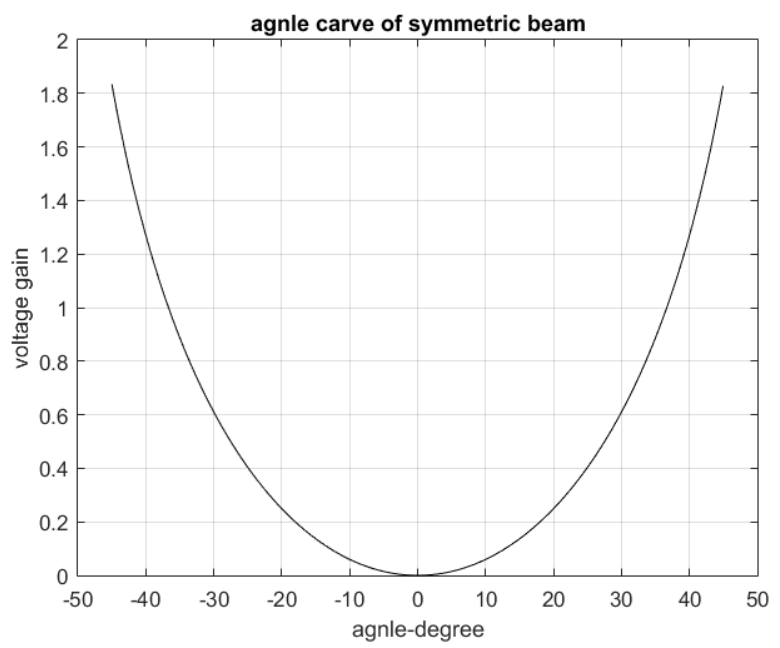

Figure. 5 The angle carve of the symmetrical beam monopulse based on uniform circular array

\subsection{Effect of thermal noise on symmetric beam pattern monopulse}

Generally, the precision of angle measurement is affected by the thermal noise. It is assumed that the thermal noise of the sum channel and the difference channel are $n_{\Delta}(t)$ and $n_{\Sigma}(t)$, separately. For this system, the ratio of the difference pattern to the sum pattern is:

$$
\frac{\Delta}{\Sigma}=\frac{V_{\Delta 1}\left(\theta_{d 1}\right) V_{\Delta 2}\left(\theta_{d 2}\right)\left(1+\rho^{2}\right)+n_{\Delta}(t)}{V_{\Sigma 1}\left(\theta_{d 1}\right) V_{\Sigma 2}\left(\theta_{d 2}\right)\left(1+\rho^{2}\right)+n_{\Sigma}(t)}=\frac{V_{\Delta 1}\left(\theta_{d 1}\right) V_{\Delta 2}\left(\theta_{d 2}\right)+n_{\Delta}(t) /\left(1+\rho^{2}\right)}{V_{\Sigma 1}\left(\theta_{d 1}\right) V_{\Sigma 2}\left(\theta_{d 2}\right)+n_{\Sigma}(t) /\left(1+\rho^{2}\right)}
$$

Under conditions of thermal noise exists, the ratio is a complex number. In order to eliminate the influence of thermal noise, the influence of the complex part must be eliminated. From equation (12), it can be concluded that the target only contributes to the real part of the ratio. The thermal noise contributes to both the real part and the imaginary part. So we take the real part of the ratio for elevation angle estimation. In this way, the influence of thermal noise is reduced and the the precision of angle measurement is improved.

\subsection{The influence of asymmetrical specular reflection}

In Figure. 1, we suppose that which can be satisfied $\theta_{i}=-\theta_{d}$, when target is far away from radar. In practice, the incident angle of specular reflection signal is slightly larger than that of the direct signal, which can be denoted as $\theta_{i}=-\left(\theta_{d}+\Delta \theta\right)$. Then the monopulse ratio is:

$$
\begin{aligned}
\frac{\Delta}{\Sigma} & =\frac{V_{\Delta 1}\left(\theta_{d 1}\right) V_{\Delta 2}\left(\theta_{d 2}\right)+\rho^{2} V_{\Delta 1}\left(\theta_{d 1}+\Delta \theta\right) V_{\Delta 2}\left(\theta_{d 2}+\Delta \theta\right)}{V_{\Sigma 1}\left(\theta_{d 1}\right) V_{\Sigma 2}\left(\theta_{d 2}\right)+\rho^{2} V_{\Sigma 1}\left(\theta_{d 1}+\Delta \theta\right) V_{\Sigma 2}\left(\theta_{d 2}+\Delta \theta\right)}=\frac{V_{\Delta 1}\left(\theta_{d 1}\right) V_{\Delta 2}\left(\theta_{d 2}\right)+\rho^{2} V_{\Delta 1}\left(\theta_{d 1}+\Delta \theta\right) V_{\Delta 2}\left(\theta_{d 2}+\Delta \theta\right)}{V_{\Sigma 1}\left(\theta_{d 1}\right) V_{\Sigma 2}\left(\theta_{d 2}\right)+\rho^{2} V_{\Sigma 1}\left(\theta_{d 1}+\Delta \theta\right) V_{\Sigma 2}\left(\theta_{d 2}+\Delta \theta\right)} \\
& =\frac{V_{\Delta 1}\left(\theta_{d 1}\right) V_{\Delta 2}\left(\theta_{d 2}\right)+\rho^{2}\left[V_{\Delta 1}\left(\theta_{d 1}\right)+V_{\Delta 1}^{\prime}\left(\theta_{d 1}\right) \Delta \theta\right] \cdot\left[V_{\Delta 2}\left(\theta_{d 2}\right)+V^{2}{ }_{\Delta 2}\left(\theta_{d 2}\right) \Delta \theta\right]}{V_{\Sigma 1}\left(\theta_{d 1}\right) V_{\Sigma 2}\left(\theta_{d 2}\right)+\rho^{2}\left[V_{\Sigma 1}\left(\theta_{d 1}\right)+V_{\Sigma 1}^{\prime}\left(\theta_{d 1}\right) \Delta \theta\right]\left[V_{\Sigma 2}\left(\theta_{d 2}\right)+V_{\Sigma 2}^{\prime}\left(\theta_{d 2}\right) \Delta \theta\right]}
\end{aligned}
$$

Generally the $\Delta \theta$ is very small, so the monopulse ratio can be approximated as:

$$
\frac{\Delta}{\Sigma} \approx \frac{V_{\Delta 1}\left(\theta_{d 1}\right) V_{\Delta 2}\left(\theta_{d 2}\right)\left(1+\rho^{2}\right)}{V_{\Sigma 1}\left(\theta_{d 1}\right) V_{\Sigma 2}\left(\theta_{d 2}\right)\left(1+\rho^{2}\right)}=\frac{V_{\Delta 1}\left(\theta_{d 1}\right) V_{\Delta 2}\left(\theta_{d 2}\right)}{V_{\Sigma 1}\left(\theta_{d 1}\right) V_{\Sigma 2}\left(\theta_{d 2}\right)}
$$

Therefore, the error caused by it asymmetrical specular reflection can be ignored. 


\subsection{The relationship between angle measurement performance and SNR}

Assume that the incident angle is 30 degrees, the reflection coefficient is $1+\mathrm{j}$. Through 1024 Mont Carlo simulations, the root mean square (RMSE) of the error varies with the signal-to-noise ratio (SNR) is illustrated in Fig. 6. It can be seen that the error decreases with the increase of SNR, which is consistent with the theoretical analysis.

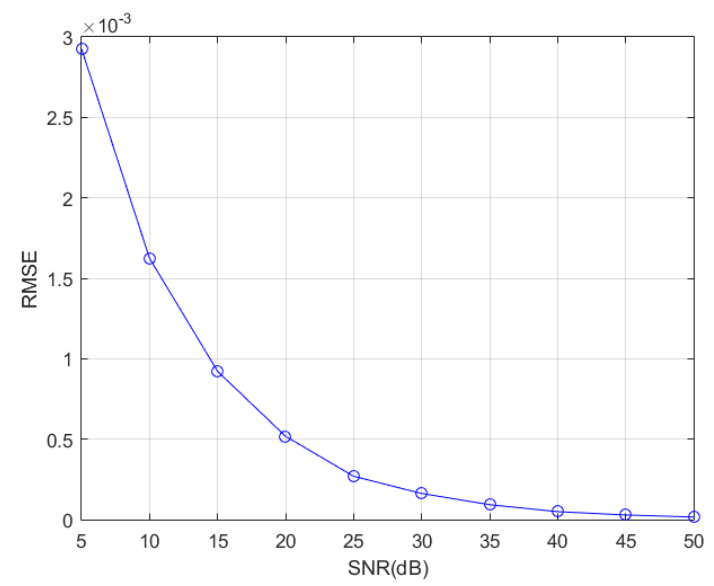

Figure. 6 The relationship RMSE and SNR

\subsection{The relationship between angle measurement performance and the reflection coefficient}

Assume that the SNR is $30 \mathrm{~dB}$, and the incident angle is 30 degrees. In the simulation, the root mean square (RMSE) of the error varies with the amplitude of the complex reflection coefficient $\left|1+\rho^{2}\right|$ as shown in Fig. 7. The error decreases with the increase of $\left|1+\rho^{2}\right|$.

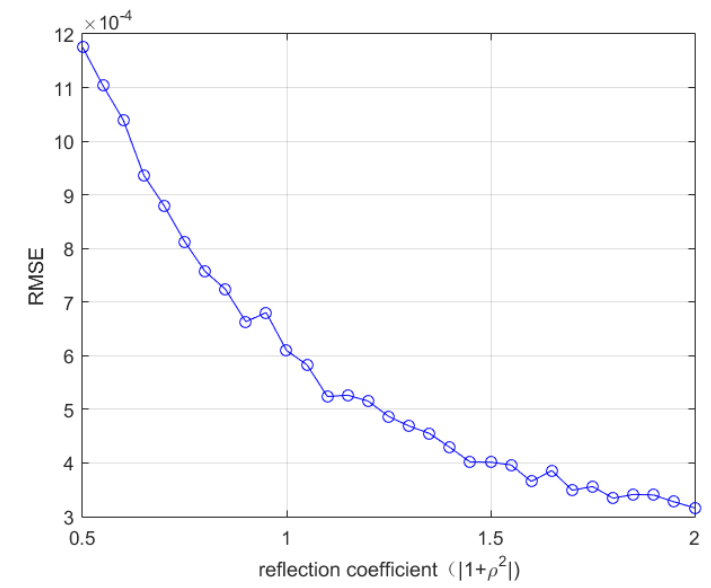

Figure. 7 The relationship RMSE and $\left|1+\rho^{2}\right|$

\section{Conclusions}

In this paper, the traditional symmetrical beam pattern monopulse is applied to the uniform circular array. We divide the array into two sets of conventional monopulse system, and then obtain the response curve of the entire system by multiplying the response curves of two monopulse system. The angle curve of the symmetrical beam and the angular resolution are analyzed by simulation. Moreover, the negative effects of thermal noise, asymmetrical multipath, SNR and 
complex reflection coefficient on angle measurement performance are discussed by simulation. Theoretical analysis and simulation results verify the feasibility of this method.

\section{References}

[1] Zhenhai Xu; Ziyuan Xiong; Jiani Wu; Shunping Xiao. Symmetrical difference pattern monopulse for low-angle tracking with array radar. IEEE Transactions on Aerospace and Electronic Systems. Year: 2016, Volume: 52, Issue: 6

[2] Sang-Gyu Kim; Kai Chang . Low-cost monopulse antenna using bi-directionally-fed microstrip patch array. Electronics Letters. Year: 2003, Volume: 39, Issue: 20

[3] Zhenjie Zhang; Zhengjun Li. Design of array fed imaging reflector antenna for monopulse limited scan use. 2010 International Conference on Microwave and Millimeter Wave Technology.Year: 2010

[4] Guo Ying; Wang Bu-hong. Effective array calibration criterions for radar low-angle tracking. IEEE Antennas and Propagation Society International Symposium. Year: 2005, Volume: 2B

[5] L. Sakr. The higher order modes in the feeds of the satellite monopulse tracking antennas. 11th IEEE Mediterranean Electrotechnical Conference. Year: 2002

[6] W. D. White. Low-Angle Radar Tracking in the Presence of Multipath. IEEE Transactions on Aerospace and Electronic Systems. Year: 1974, Volume: AES-10, Issue: 6

[7] M. A. Sebt; A. Sheikhi; M. M. Nayebi. Robust low-angle estimation by an array radar. IET Radar, Sonar \& Navigation. Year: 2010, Volume: 4, Issue: 6 